\title{
Tree diversity in western Kenya: using profiles to characterise richness and evenness
}

\author{
R. KINDT ${ }^{1, *}$, P. VAN DAMME ${ }^{2}$ and A.J. SIMONS ${ }^{1}$ \\ ${ }^{1}$ ICRAF, PO Box 30677-00100, Nairobi, Kenya; ${ }^{2}$ Ghent University, Laboratory of Tropical and \\ Subtropical Agriculture and Ethnobotany, Coupure Links 653, B-9000 Gent, Belgium; * Author for \\ correspondence (e-mail: R.Kindt@CGIAR.org; phone: 254-2-524000 or 1-650-8336645; fax: 254-2- \\ 524001 or $1-650-8336646)$
}

Received 18 May 2004; accepted in revised form 7 January 2005

Key words: Accumulation, Agroforestry, Diversification, Domestication, Evenness, Rarefaction, Rényi diversity profile, Richness

Abstract. Species diversity is a function of the number of species and the evenness in the abundance of the component species. We calculated diversity and evenness profiles, which allowed comparing the diversity and evenness of communities. We applied the methodology to investigate differences in diversity among the main functions of trees on western Kenyan farms. Many use-groups (all trees and species that provide a specific use) could not be ranked in diversity or evenness. No usegroup had perfectly even distributions. Evenness could especially be enhanced for construction materials, fruit, ornamental, firewood, timber and medicine, which included some of the most species-rich groups of the investigated landscape. When considering only the evenness in the distribution of the dominant species, timber, medicine, fruit and beverage ranked lowest ( $>60 \%$ of trees belonged to the dominant species of these groups). These are also use-groups that are mainly grown by farmers to provide cash through sales. Since not all communities can be ranked in diversity, studies that attempt to order communities in diversity should not base the ordering on a single index, or even a combination of several indices, but use techniques developed for diversity ordering such as the Rényi diversity profile. The rarefaction of diversity profiles described in this article could be used in studies that compare results from surveys with different sample sizes.

\section{Introduction}

One of the objectives of tree domestication research, in general and more specific, in western Kenya is the diversification of tree species composition in agroecosystems (Kindt and Lengkeek 1999; Kindt et al. 2004). In the realm of agroforestry, underpinning the need for diversification is the desire to enhance the stability and productivity of agroecosystems (ICRAF 1997; Atta-Krah et al. 2004).

Diversity means different things to different people. Most often in natural or agricultural systems, species counts (species richness) are provided as the measure of diversity. Continuing this logic, diversification means adding more species. Species diversity, however, is a function of the number of species, and the evenness in distribution of species' abundances (Magurran 1988; Purvis and Hector 2000). Options for diversification can therefore be dissociated into interventions that target richness and those that target evenness. 
We investigated the diversity of various groups of trees that contributed to similar service or production functions on farms in western Kenya, as our target was diversification within these functions, and not of overall diversity. Since heterogeneity in characteristics of species results in saturating effects of increased diversity on ecosystem functioning (e.g., Hector et al. 1999; Loreau et al. 2001; Tilman et al. 2001), we expect larger effects from increasing diversity within communities of lower diversity. Ranking of use-groups in diversity, therefore, allows prioritising their scope for diversification measured as the average expected effect of adding one species. In addition, such approaches provide the opportunity to model effects of replacement, substitution and expansion at fixed and varying tree densities.

Appropriate techniques for measuring diversity and evenness were used in this article. Single indices of diversity or evenness can result in wrong interpretation since not all communities can be ranked in diversity or evenness, whereas they can always be ranked on the basis of a single index (Taillie 1979; Tóthmérész 1995; Ricotta and Avena 2002; Ricotta 2003). Since diversity research should not be based on single indices of diversity or evenness, techniques for diversity and evenness ordering were used that produce diversity and evenness profiles.

\section{Material and methods}

Study area

Complete tree inventories were made on 201 stratified-randomly selected farms (taken to mean all land managed by a single household) in the Vihiga and Kakamega districts of western Kenya. The study area is inhabited predominantly by the Luhya (Luyia) ethnic group and belongs to the same agroecological zone where altitude ranges $1500-1800 \mathrm{~m}$ above sea level, annual mean temperature ranges $18.1-20.4{ }^{\circ} \mathrm{C}$, and annual bimodal rainfall ranges 1600 $2000 \mathrm{~mm}$ (Jaetzhold 1982). Four villages were selected within the area, each located in a different stratum that mainly differed in farm sizes and arrangement of woody biomass in the landscape (Bradley et al. 1985; Bradley 1991). The selection of villages coincided with a gradient towards the species-rich Kakamega Forest National Reserve.

\section{Information recorded on tree species}

All trees (woody perennials) were censused using Beentje (1994) as the key reference. For each tree species encountered on a farm, its abundance (the total number of trees) and uses (see below) were recorded by participatory interviews with household informants involving farm walks, tree counting by the interviewer and data recording on a species-by-species basis. 
Households listed all the products or services (uses) that are provided by the different species encountered on their farm. Free responses were obtained on tree uses (primary and all secondary uses) that were postcoded during data entry and checking. In total, 60 use categories were recorded, but analyses were only conducted for the 12 use categories that occurred on more than $20 \%$ of farms. Because it is possible that some informants could have forgotten some uses of particular species, information was adjusted (increasing species-farm-use combinations from 6859 to 7526 ) by always including all trees of a species in a use-group if more than $50 \%$ of farmers and minimum five farmers with the species mentioned the use.

Use-groups were defined as all the trees encountered in the survey that provided one particular type of use. These use-groups were analysed by matrices with as rows the information collected on a particular farm and as columns the various species encountered. Each cell of a use-group matrix provides the number of trees of each species that was used for a particular function on a particular farm.

\section{Diversity and evenness profiles}

The Rényi diversity profile is one of the techniques for diversity ordering that were specifically designed to rank communities from low to high diversity. Rényi diversity profile values $\left(H_{\alpha}\right)$ are calculated from the frequencies of each component species (proportional abundances $p_{i}=$ abundance of species $i /$ total abundance) and a scale parameter $(\alpha)$ ranging from zero to infinity (Tóthmérész 1995; Legendre and Legendre 1998) as:

$$
H_{\alpha}=\frac{\ln \left(\sum p_{i}^{\alpha}\right)}{1-\alpha}
$$

It can be demonstrated that values of the Rényi profile at the respective scales of $0,1,2$ and $\infty$ are related to species richness $S$, the Shannon diversity index $H$, the Simpson diversity index $D^{-1}$ and the Berger-Parker diversity index $d^{-1}$ (Magurran 1988; Legendre and Legendre 1998; Shaw 2003):

$$
\begin{gathered}
H_{0}=\ln (S) \\
H_{1}=H=-\sum p_{i} \log p_{i} \\
H_{2}=\ln \left(D^{-1}\right)=\ln \left(\sum\left(p_{i}^{2}\right)^{-1}\right) \\
H_{\infty}=\ln \left(d^{-1}\right)=\ln \left(p_{\max }^{-1}\right)
\end{gathered}
$$


Community $A$ is more diverse than a community $B$ if the diversity profile for community $A$ is everywhere above the diversity profile for community $B$. Communities that have intersecting profiles cannot be ordered in diversity. The fact that intersecting profiles (partial diversity ordering) could occur explains why ordering techniques such as the Rényi series are needed, since a single diversity index will not provide sufficient information. The values of the series for the various use-groups were calculated for scales $\alpha \in\{0,0.25,0.5,1,2,4,8$, $\infty$.

From the diversity profile we derived an evenness profile $\left(\ln E_{\alpha, 0}\right)$ that orders communities in evenness in a similar way that diversity profiles order communities in diversity (Kindt et al. 2001; Ricotta and Avena 2002):

$$
\ln E_{\alpha, 0}=H_{\alpha}-H_{0}
$$

Partial ordering may occur where evenness profiles intersect, indicating that intrinsic evenness ordering is not possible. For example, community $A$ with species abundances of $(70,20,10)$ can only be partially ordered with community $B$ with species abundances of $(60,35,5)$ and it is therefore not possible to identify the community of largest evenness. The example above is a rare case where diversity ordering equals evenness ordering since species richness is equal in community $A$ and $B$. Since species richness differs between most communities, diversity and evenness ordering can only be inferred through separate ordering techniques that conform to diversity ordering (such as the Rényi diversity series) or to evenness ordering (such as Hill's $E_{\alpha, 0}$ subfamily, see below).

Since $H_{0}=\ln S$ (as shown above), rearranging the above formula shows that the Rényi diversity profile can be decomposed as:

$$
H_{\alpha}=\ln S+\ln E_{\alpha, 0}
$$

This decomposition has the attractive feature in showing mathematically that diversity combines information on species richness and evenness, which conforms to the definition of diversity (Magurran 1988; Purvis and Hector 2000; Shaw 2003). The decomposition of the Rényi profile is a generalisation of the decomposition of the Shannon index $H_{1}$ as $\ln S+\ln E$ (Hayek and Buzas 1997; Kindt et al. 2001).

$E_{\alpha, 0}$ is a subfamily of Hill's parametric evenness (Hill 1973; Ricotta and Avena 2002; Ricotta 2003) defined as:

$$
E_{\alpha, 0}=\frac{N_{\alpha}}{N_{0}}=\frac{\exp H_{\alpha}}{\exp H_{0}}
$$

$E_{\alpha, 0}$ has several desirable properties as an evenness function: it is consistent with the Lorenz ordering and therefore meets the general requirements for an evenness index (Taillie 1979; Rousseau et al. 1999; Ricotta 2003), it is formally related to diversity (Ricotta and Avena 2002; Ricotta 2003), and it is normalised between zero and one. 
Since $\ln E_{\alpha, 0}$ is a monotone transformation of $E_{\alpha, 0}$, both these measures provide the same rank order when communities are compared for evenness despite the fact that $\ln E_{\alpha, 0}$ is not normalised between zero and one. In analogy with diversity profiles (Tóthmérész 1995), the various techniques provide the same ordering, but differ in the resolution of the graphs that they produce.

\section{Accumulation patterns for the Rényi series}

We calculated the Rényi diversity profile for each use-group by calculating the frequencies of each species in the entire survey. These profiles allow comparing the total diversity of the various use-groups at the scale of the total survey. Since some use-groups did not occur on each farm, another comparison was made by only including farms where a specific use-group occurred. Because the total number of farms per use-group differed, profiles were rarefied to the same number of farms (the number of farms of the smallest use-group) so that effects from differences in sample size could be removed from the analysis.

The rarefaction of the diversity profile was achieved through a Monte-Carlo approach of 1000 random additions of farms (selecting the first farm at random, adding the second farm at random,...) using sampling without replacement. We obtained accumulation surfaces for diversity profiles by calculating the average $H_{\alpha}$ for each subset of $1,2, \ldots$, all farms combined, and for each $\alpha \in\{0,0.25,0.5,1,2,4,8, \infty\}$ (Kindt et al. 2001). A similar approach of rarefaction is used to calculate species accumulation curves for random pooling of sample units (e.g., Colwell 1997; Gotelli and Colwell 2001). FORTRAN and R statistical programmes developed to carry out the computations can be obtained from the authors (Kindt 2001, 2004).

$H_{\infty}$ was the only value for which $95 \%$ confidence interval (CI) limits for the expected values for the entire survey area could be calculated as it is obtained solely from the proportion of the dominant species, while other values in the diversity series include an effect of species richness. Hayek and Buzas (1997) propose to calculate the variance of species frequency $(p)$ in the case of cluster sampling as

$$
\widehat{\sigma}_{p c l u s}^{2}=\frac{\sum_{i=1}^{n} m_{i}^{2}\left(p_{i}-p\right)^{2}}{(n-1) m^{2} n^{-1}}
$$

( $m_{i}=$ total abundance of farm $i, p_{i}=$ species frequency in farm $i, p=$ species frequency of the total survey, $n=$ number of farms, and $m=$ total abundance). $95 \%$ CI limits for the proportion of the dominant species can then be calculated as:

$$
p \pm t \cdot \widehat{\sigma}_{p c l u s}
$$


The limits of the CI for the proportion were $y=\ln \left(x^{-1}\right)$ transformed to calculate a CI for $H_{\infty}$.

\section{Results}

Diversity ordering and examination of richness and evenness contributions

Figure 1 shows the Rényi diversity ordering of all trees and the 12 most frequent use-groups. By examining the values at scales $0,1,2$, and $\infty$, species richness and values of Shannon, Simpson, and Berger-Parker diversity indices can be inferred. The many intersections in the figure show the difficulties to order most groups in diversity. Figure 2 shows many groups with intersecting evenness profiles, which is an indication that many groups cannot be ranked in evenness. Table 1 provides some more precise statistics for certain profile values than can be inferred from the figures, and also gives some parameters that describe the pattern of the various diversity profiles (see below).

We differentiated between five pools of use-groups based on their total species richness $\left(H_{0}\right)$ (which is an ordering of use-group only based on richness): (i) pool A - very high richness, including all trees and firewood; (ii) pool $\mathrm{B}$ - high richness, including shade; (iii) pool $\mathrm{C}$ - medium richness, including

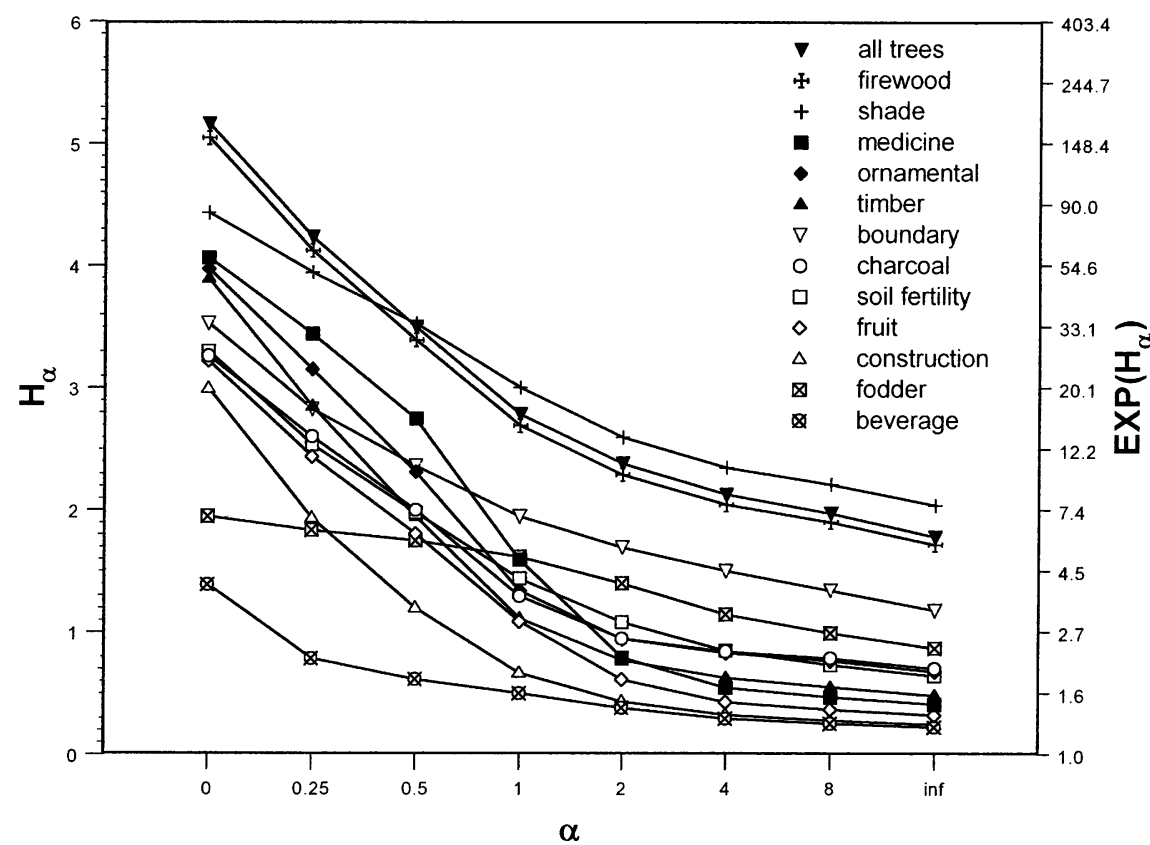

Figure 1. Diversity profiles based on the Rényi series $H_{\alpha}$ for all trees and trees belonging to particular use-groups. 


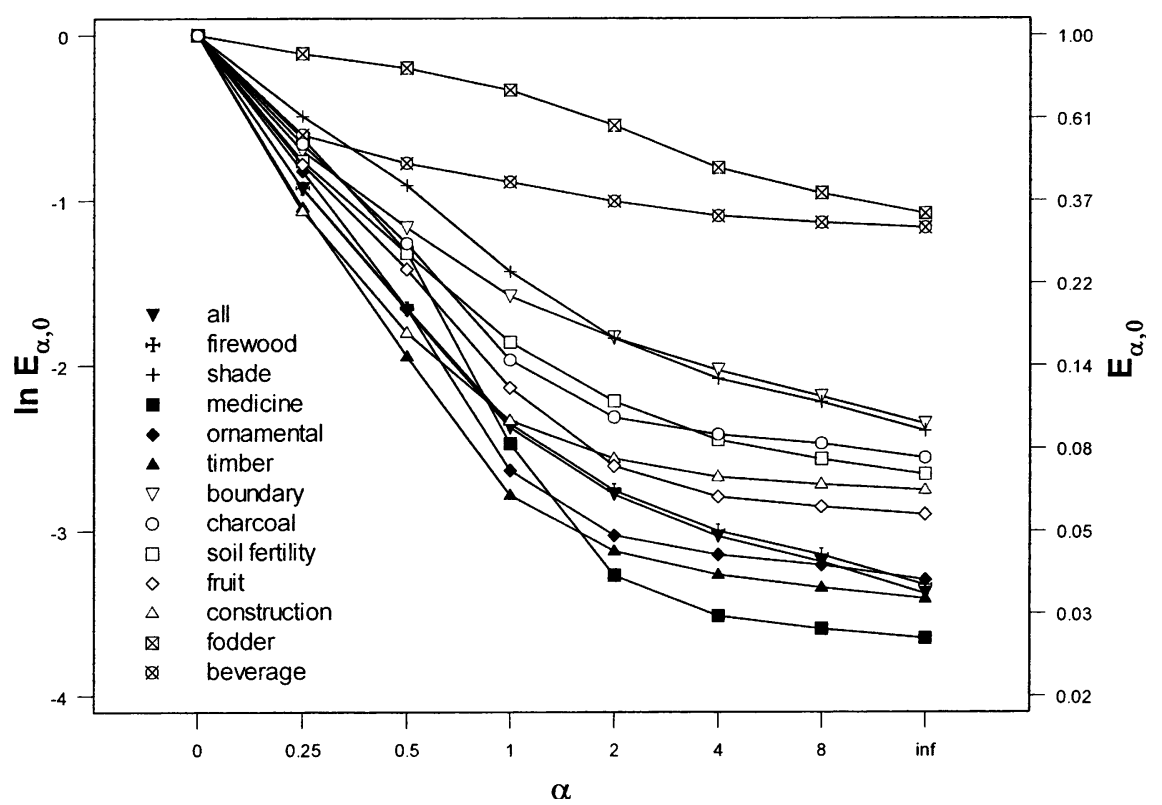

Figure 2. Evenness profiles for all trees and trees belonging to particular use-groups.

Table 1. Values for specific profile values of the diversity and evenness profiles depicted in Figures 1 and 2 with for all trees and for the most frequent use-groups in western Kenya.

\begin{tabular}{lllllllll}
\hline Use-group & $H_{0}$ & $H_{1}$ & $H_{2}$ & $H_{\infty}$ & $\ln E_{1,0}$ & $E_{1,0}$ & $H_{\infty}, I$ & $H_{\infty}, u$ \\
\hline All trees & 5.16 & 2.79 & 2.38 & 1.78 & -2.37 & 0.09 & 1.61 & 1.98 \\
Firewood & 5.04 & 2.69 & 2.29 & 1.71 & -2.35 & 0.10 & 1.54 & 1.92 \\
Shade & 4.43 & 3.00 & 2.60 & 2.04 & -1.43 & 0.24 & 1.43 & 3.84 \\
Medicine & 4.06 & 1.59 & 0.79 & 0.41 & -2.47 & 0.08 & 0.15 & 0.77 \\
Ornamental & 3.97 & 1.34 & 0.94 & 0.68 & -2.63 & 0.07 & 0.37 & 1.12 \\
Timber & 3.89 & 1.11 & 0.77 & 0.48 & -2.78 & 0.06 & 0.36 & 0.61 \\
Boundary & 3.53 & 1.95 & 1.69 & 1.17 & -1.58 & 0.21 & 0.97 & 1.44 \\
Soil fertility & 3.30 & 1.44 & 1.08 & 0.64 & -1.86 & 0.16 & 0.17 & 1.57 \\
Charcoal & 3.26 & 1.29 & 0.95 & 0.70 & -1.97 & 0.14 & 0.29 & 1.41 \\
Fruit & 3.22 & 1.08 & 0.61 & 0.32 & -2.13 & 0.12 & 0.24 & 0.41 \\
Construction & 3.00 & 0.66 & 0.43 & 0.24 & -2.33 & 0.10 & 0.17 & 0.32 \\
Fodder & 1.95 & 1.61 & 1.40 & 0.87 & -0.33 & 0.72 & 0.44 & 1.63 \\
Beverage & 1.39 & 0.50 & 0.38 & 0.22 & -0.89 & 0.41 & 0.04 & 0.44 \\
\hline
\end{tabular}

$H_{\infty} ; l$ and $H_{\infty} ; u$ are $95 \%$ CI limits for $H_{\infty}$. See methods for the formulas.

medicine, ornamental and timber; (iv) pool D - moderate richness, including boundary demarcation, soil fertility enhancement, charcoal, fruit and construction wood; and (v) pool E - low richness, including fodder and beverage. Some differences and similarities in diversity and evenness can subsequently be detected between and within pools (Figures 1 and 2). 
When comparing diversity and evenness patterns for pools, it can be observed that use-groups of pool A and B were more diverse than the other use-groups. The evenness profile (Figure 2) shows that shade (pool B) has a more even distribution than use-groups of pool A. Diversity profiles of use-groups of pool C intersected all use-groups of pool D and E, with the exception of fruit and construction (pool D) and beverage (pool E). The evenness profiles, however, show that ornamental and timber (pool C) had lower evenness than all use-groups of pool $\mathrm{D}$, with exception of construction for which intersection can be observed. Fodder and beverage (pool E) had the most even distribution of all use-groups, with the exception of an intersection for beverage and shade.

When comparing diversity for use-groups that belong to the same pool, it can be seen that all trees are more diverse but that firewood is more even within pool A although the profiles were almost parallel to each other. The evenness profile of medicine intersected the other use-groups of pool C. All use-groups of pool $\mathrm{C}$ had intersecting evenness profiles. These groups can therefore not be ranked in evenness within pool C. Most use-groups within pool D could be ranked in diversity, since their diversity profiles did not intersect. Boundary demarcation is the most diverse and evenly distributed use-group within the pool.

Construction is the least diverse use-group and fruit the second least diverse of pool D. Their evenness profiles intersect, however. Charcoal and soil fertility had intersecting diversity and evenness profiles. Within pool E, fodder is more diverse and more evenly distributed.

Values of $H_{\infty}$ (Figure 1 and Table 1) lower than 0.5 indicate that the dominant species of timber, medicine, fruit, construction and beverage contains more than $60 \%$ of all trees of these use-groups. Values of $H_{\infty}$ between 0.5 and 1.5 indicate that the dominant species contains between 22 and $60 \%$ of all trees for soil fertility, charcoal, ornamental, fodder and boundary demarcation. The dominant species contains a smaller percentage of trees for the other usegroups. However, values of $\ln E_{\infty, 0}$ (Figure 2) show that the dominant species for all trees and firewood are less evenly distributed when comparing with many other use-groups (the formula $\ln E_{\infty, 0}=H_{\infty}-H_{0}$ implies that when species richness increases, the frequency of the dominant species should decrease to maintain the same $\left.\ln E_{\infty, 0}\right)$. Medicine had the least evenly distributed dominant species.

\section{Effects of sample size on diversity}

Figure 3 shows the average profile values and associated 95\% CI for subsamples of 47 farms (the number of farms with fodder, the least frequent usegroup). Figure 3 indicates that, on average, similar profiles are obtained as for the full sample. Diversity profiles, especially medicine and shade, had large 95\% CI, however. The 95\% CI still allow classifying use-groups in the richness 
pools that we differentiated above. Construction of the original pool D obtained richness values closer to the range of pool E. Within pools, most $95 \% \mathrm{CI}$ of diversity profile values overlap, although the averages showed a similar pattern in Figures 1 and 3.

Figures 4-6 show that several use-groups (especially all trees, firewood, boundary, timber, fruit and construction) obtain stable (asymptotic) values for $H_{1}, H_{2}$ and $H_{\infty}$ after 40 farms were sampled. Some other use-groups showed some strong non-linear patterns throughout the range of pooled farms. These use-groups were especially shade, fodder, medicine, and soil fertility. Whereas the non-linear patterns were for the larger part increments of the index with increasing sample size, a declining pattern could be observed for medicine for $\mathrm{H}_{2}$ and $H_{\infty}$. The observed patterns suggest that to extrapolate profile values, in some cases (asymptotic patterns) similar values can be expected, whereas in other cases different values would be expected (non-linear patterns). However, extrapolation should be used carefully since we had no actual data for larger sample sizes.

Table $1\left(H_{\infty, l}\right.$ and $\left.H_{\infty, u}\right)$ indicates the $\mathrm{CI}$ that is expected for the average value of $H_{\infty}$ when all farms would be sampled in the survey area (assuming random sampling of the farms). Relatively large CI were even obtained for

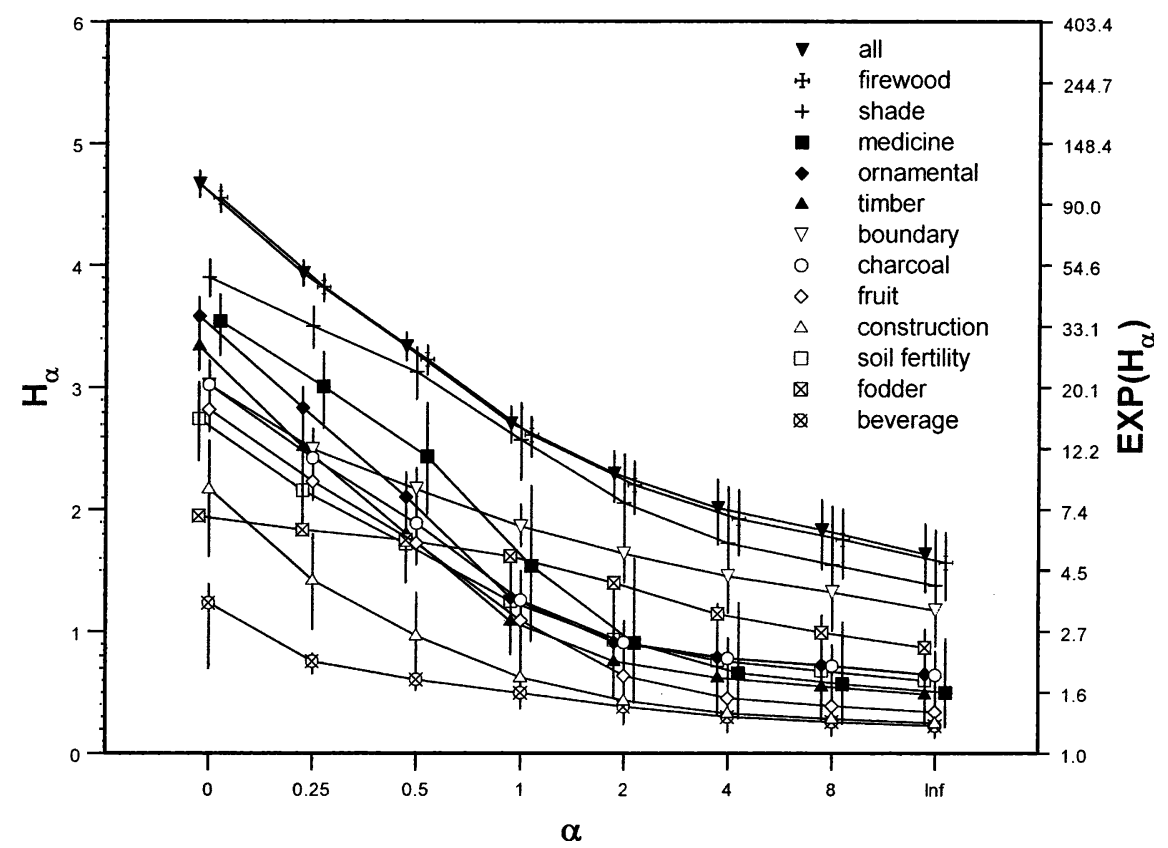

Figure 3. Averages of rarefied diversity profiles based on the Rényi series $H_{\alpha}$ for all trees and trees belonging to particular use-groups calculated from 1000 random subsamples of 47 farms, with $95 \%$ CI. All profile values were calculated for the same scales, but groups were presented at different scales for better discrimination. 


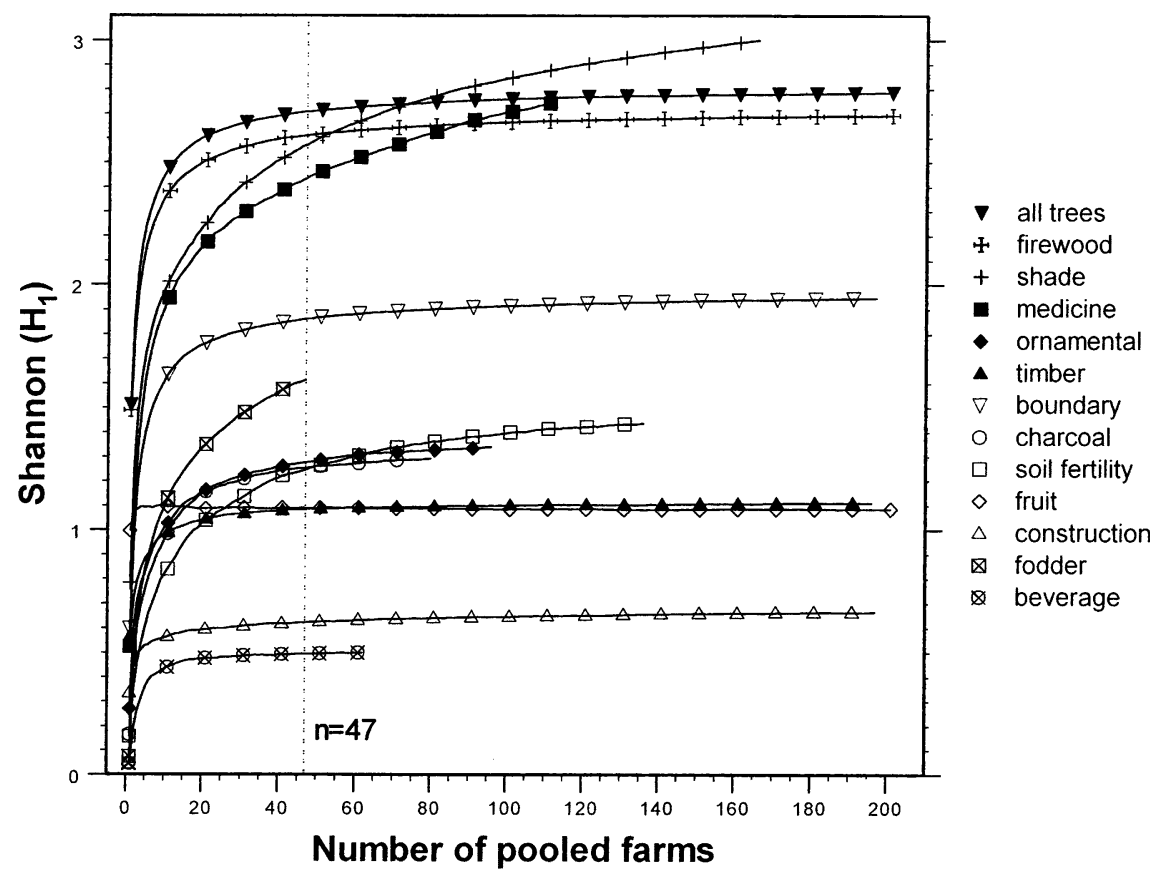

Figure 4. Accumulation curve for the Shannon diversity index for all trees and trees belonging to particular use-groups. The vertical reference indicates the sample size of 47 farms depicted in Figure 3.

groups where stable values were obtained at the respective scale in Figure 6 (for example timber, fruit, construction, and beverage). However, these CI were among the smallest. Large CI corresponded to non-linear patterns for the Berger-Parker index (Figure 6, see discussion above). Medicine which had a non-linear negative pattern for the Berger-Parker index had a relatively small $\mathrm{CI}$, however.

\section{Discussion}

Using diversity and evenness profiles

We compared the diversity of various use-groups by using the Rényi diversity profile. The numerous intersections of diversity profiles indicate situations of partial ordering in our data set. If we would have used a single diversity index such as the Shannon, Simpson or Berger-Parker index, then we have obtained erroneous results. Studies that attempt to order communities in diversity (e.g., Dougall and Dodd 1997; Slik et al. 2002; Mishra et al. 2004; Zilihona et al. 2004) should therefore not base the ordering on a single index, or even a 


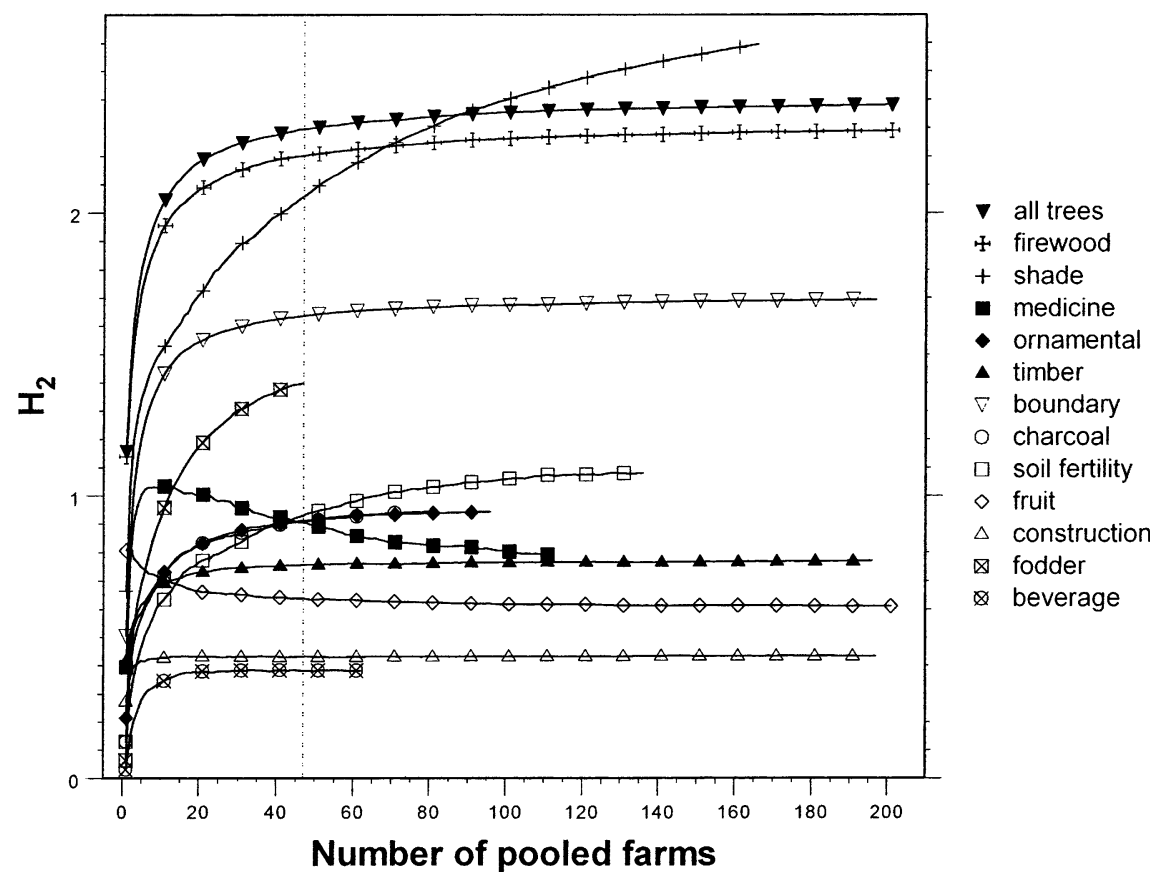

Figure 5. Accumulation curve for $\mathrm{H}_{2}$ for all trees and trees belonging to particular use-groups. The vertical reference indicates the sample size of 47 farms depicted in Figure 3.

combination of several indices, but use techniques developed for diversity ordering such as the Rényi diversity profile. Although the aim of our study was not to compare the performance of several diversity ordering techniques, we agree with Tóthmérész (1995) that the Rényi profile is one of the most useful methods for diversity ordering.

Various researchers have used models of rank-abundance curves to study biodiversity (e.g., Magurran 1988; Hayek and Buzas 1997; Hubbell 2001; Belaoussoff et al. 2003; Magurran and Henderson 2003; McGill 2003). Because rank-abundance curves do not provide a direct graphical method for diversity ordering (some diversity ordering techniques are based on cumulative frequencies, but none use the frequencies of the individual species; Tóthmérész 1995), and since information is lost when rank-abundance curves are modelled, we do not recommend using (models of) rank-abundance distribution for diversity ordering.

As for the diversity profile, we observed various intersections in the evenness profiles for our dataset. For this reason, a single index of evenness (e.g., Zilihona et al. 2004) is not sufficient information to order communities in evenness in the same way that a single index of diversity is not sufficient for diversity ordering. 


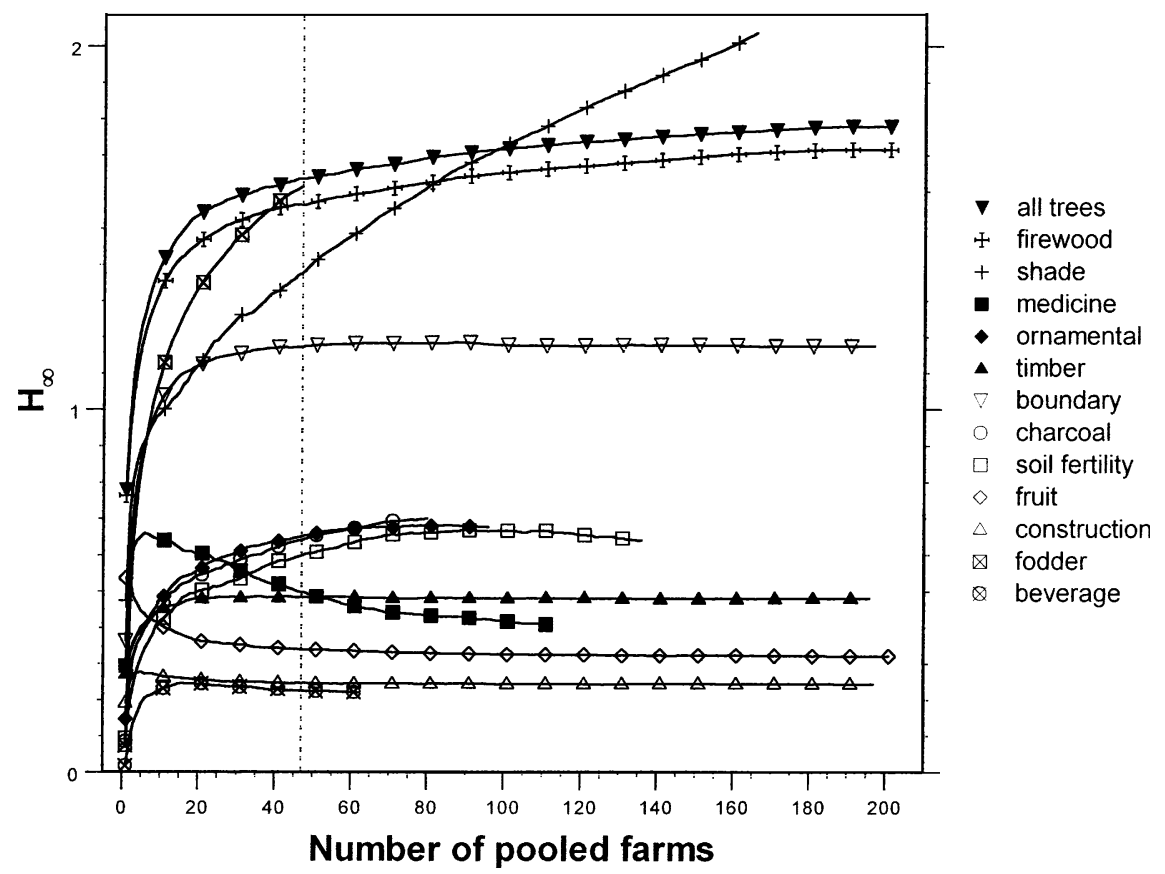

Figure 6. Accumulation curve for $H_{\infty}$ for all trees and trees belonging to particular use-groups. The vertical reference indicates the sample size of 47 farms depicted in Figure 3.

The influence of sample size on the diversity profile

It has been observed for a long time in ecological research that sample size has an influence on species richness (e.g., Arrhenius 1921). Since diversity is influenced by richness, sample size also has an effect on diversity. We were able to investigate the influence of sample size on diversity by studying the range of values observed in subsets of the data by using a randomisation approach similar to the randomisation approach to calculate species accumulation curves (Kindt et al. 2001). This approach allowed rarefaction to the same number of sample units (farms in our example), thus removing the effects from differences in sample size. This approach could be useful in other studies that compare results obtained from surveys with different sample sizes, especially since the approach includes a diversity ordering technique which is necessary for an accurate description of the diversity of a community.

Magurran (1988) indicated that a Rényi series value with larger scale parameter value has reduced sensitivity to sample size. Gimaret-Carpentier et al. (1998) observed that the Simpson index reached stable values at lower sample sizes compared to the Shannon index.

We observed such asymptotic patterns both for $H_{2}$ and $H_{\infty}$ for those groups where stable values were obtained for the Shannon index (timber, fruit, 
construction, and beverage). Shade and fodder, however, showed the other extreme with increasing profile values with increasing sample size, and not asymptotic values.

Hayek and Buzas (1997) proposed to investigate accumulation patterns of $H, \ln (E)$ and $\ln (E) / \ln (S)$ to choose the best abundance distribution model ('SHE analysis'). Reaching asymptotic values for these statistics with increasing sample size would indicate that the species abundance distribution corresponds to respectively a log-series, a broken-stick or log-normal distribution. Based on our results, we could therefore conclude that most usegroups (and especially timber, fruit, construction and beverage) have abundance distributions more typical of the log series distribution (asymptotic $H$ ), while the distributions of shade and fodder corresponded more to a lognormal distribution (asymptotic $\ln (E) / \ln (S)$, figure not included). (A preliminary analysis found that shade, medicine and ornamental conformed to the log-normal distribution, whereas all trees, firewood, timber, and boundary did not - for other use-groups, unreliable results were obtained for chisquare goodness-of-fit tests.) We suggest expanding the SHE analysis to the complete diversity profile, as in Figure 3. For example, for a pure log-series (and geometric series), the Simpson and Berger-Parker indices are also expected to reach constant values (May 1975), which was a phenomenon that we could observe most clearly for the groups with the clearest asymptotic $H_{1}$ pattern. Since asymptotic $H_{1}$ and $H_{2}$ are only expected for the log-series distribution, the findings of Gimaret-Carpentier et al. (1998) described above most likely correspond to studies of systems more conform to this distribution. For the same reason, the observation of reduced sensitivity to sample size with increasing scale provided by Magurran (1988) could only apply to the $\log$-series.

\section{Planning diversification based on diversity profiles}

The basic aim of our study was to plan for diversification. The diversity and evenness profiles were calculated on several use-groups. By differentiating between several use-groups, use-groups of lower diversity or evenness were identified and a benchmark dataset was created to measure the impact of future diversification. By identifying use-groups of lower relative diversity (such as beverage and construction), priority can be given to these use-groups for diversification. Such approach can be described as a coldspot approach that focuses on subregions of lowest diversity in a study area. For example, it would be less efficient for diversification to add new species to firewood than adding new species to construction. With the rarefaction procedure, comparisons are made only for farmers that are already growing trees for a use-group. This approach should be preferred when there is no scope for increasing the number of farmers for each use-group: this is a better benchmark to plan diversification efforts (see below). 
The value of using diversity profiles lays not only in determining which usegroups are more diverse. Diversity profiles also allow discrimination between richness and evenness contributions to diversity. If the intervention would attempt to increase diversity for two groups with intersecting diversity profiles, then for one group improvement of richness could be attempted, while improvement of evenness would be the target for the other group.

The lack of evenness in the distribution of the dominant species and the many steep decreases in profiles with increasing scale parameter value showed that diversity could be increased substantially in many use-groups by targeting evenness, rather than targeting richness. No group had perfectly evenly distributed species. Evenness increment could be achieved by encouraging farmers to establish trees in more even numbers (influencing the demand for tree germplasm) or by more species-even germplasm distribution (influencing the supply of tree germplasm). The analysis shows use-groups with steep diversity and evenness profiles where such diversity improvements would be most useful: i.e. the construction, fruit, ornamental (although this group will probably not constitute a priority to farmers), firewood, timber and medicine groups. When considering the frequency of the dominant species only (not how evenly this species is distributed), timber, medicine, fruit and beverage are the groups with frequencies larger than $60 \%$. Interestingly, these are also the use-groups that could be categorised as providing more cash income to farmers ('high value trees' that could be selected to be of higher priority for domestication for this reason).

The analysis of diversity did not include all aspects that could influence decisions on alterations in tree species composition on farms. Such factors include potential differences in importance attributed by farmers to different use-groups so that diversification of a more important but more diverse usegroup could be given priority over that of a less diverse but also less important use-group. Another aspect is potential differentiation among farms in alpha diversity, so that farms with low diversity for a specific use-group could be targeted, rather than only targeting use-groups with lower diversity at the survey level (Kindt et al. 2004).

Effective diversity planning will require that the relative abundances of the composing species are analysed within use-groups that were prioritised for diversification. When planning for increments in evenness, the potential for increasing the abundances of rare species should be investigated. Where it is not possible to add new trees to a particular farm or landscape, the potential of substituting some trees of the dominant species with trees of the rare species should be explored. Participatory research should investigate why some species occur in higher numbers than other species: substitution of common species by rare species may be easier when differences in abundance are not related to differences in farmer preference, but caused by factors such as differences between species in natural regeneration, historic promotion by development agencies, or erosion in local knowledge. Since all species within a particular use-group are used for that particular purpose, there is a definite potential for 
increasing evenness, but such increments may require balancing diversity with differences in preference - some species may need to be promoted for the insurance that they bring at a cost for short-term productivity.

McNeely and Scherr (2002) describe that a new type of agriculture is needed that leads to increased food security and conservation gains since human population density and biodiversity are positively correlated in many areas. Their book provides examples of innovative landscape management strategies that successfully combined both objectives by applying ecoagriculture strategies. Our study documented that many tree species have been integrated in farming systems already (conform Ecoagriculture Strategy 4 of mimicking natural habitats by integrating productive perennial plants). Since careful scrutiny of species identities of the various use-groups (such detailed study was beyond the scope for this article, but is a logical next step as described above) showed that the dominant species in most use-groups were exotic species (exceptions were boundary demarcation, fodder and medicine), whereas the majority of rare species were indigenous species, increments of evenness could result in increments of indigenous tree species in the farming landscape (since not only exotic species are planted, increasing abundances for indigenous species entails small relative changes in planting practices by local communities). Diversification could therefore result in improved conservation, although the links between development and conservation goals need to be explored carefully (Adams et al. 2004).

Landscape diversification could also consider population structure and geneflow of particular species, especially for indigenous species. Where less frequent species are promoted, interventions should attempt to ensure that population sizes are large enough to avoid substantial genetic erosion. Diversification planning could for example consider corridors in farmland between natural populations to reduce genetic erosion (O'Neill et al. 2001). Such considerations of genetic diversity may indicate limits to diversification (not all species could potentially be maintained at large enough population sizes for a more even distribution of species) that could be incorporated in planning of diversification (finding a more even species abundance distribution that avoids too small population sizes for each species, possibly with a smaller number of species). Alternatively, species can be maintained at very small population sizes given that new genetic diversity is regularly introduced from genetically diverse seed sources.

Ecological reasons for diversification within a use-group could include minimizing the chances of pest and disease outbreaks. Promotion of single species for a particular use-group should especially be avoided since several pest outbreaks on agroforestry species have been experienced after largescale promotions of monoculture agroforestry technologies (Atta-Krah et al. 2004). Ecological research has indicated that biodiversity can affect ecosystem function, but that differences in species function are conditions for positive effects of biodiversity on ecosystem stability and productivity (e.g., Hector et al. 1999; Loreau et al. 2001, 2002; Tilman et al. 2001). Natural 
communities with specific species extinction and abundance patterns function differently from experimental communities of similar richness but different composition and equalized abundances, however (Zavaleta and Hulvey 2004). The ecological consequences of increasing the diversity or evenness of trees on farms can therefore not be predicted and thus needs to be evaluated on a case-by-case basis, although natural communities can provide some clues on potential richness and composition (e.g., Van Noordwijk and Ong 1999).

Although the methods shown in this article are unlikely to provide all the answers for diversification planning, they did provide meaningful insights in relationships between richness, evenness, and sample size of use-groups. They, thus, provide accurate guidance for attempts in alterations of these characteristics. Obviously, they allow also for detailed monitoring of the impact of interventions of these characteristics by providing a baseline to compare diversity before and after interventions and by using a technique that is specifically tailored to study differences in diversity.

\section{Acknowledgements}

We are very grateful for information provided by farmers. Roeland Kindt wants to thank Wim Buysse, Richard Coe, Ian Dawson, Ard Lengkeek, Meine Van Noordwijk and two anonymous reviewers for inputs in the article, assistance provided by the ICRAF East and Central African regional programme especially through Stephen Ruigu and Amadou Niang, assistance during data collection by Joseph Njeri, and funding provided by DFID and VVOB.

\section{References}

Adams W.M., Aveling R., Brockington D., Dickson B., Elliott J., Hutton J., Roe D., Vira B. and Wolmer W. 2004. Biodiversity eradication and the eradication of poverty. Science 306: 11461149.

Arrhenius O. 1921. Species and area. J. Ecol. 9: 95-99.

Atta-Krah K., Kindt R., Skilton J.N. and Amaral W. 2004. Managing biological and genetic diversity in tropical agroforestry. Agroforest. Syst. 61: 183-194.

Beentje H.J. 1994. Kenya Trees, Shrubs and Lianas. National Museums of Kenya, Nairobi 722 pp.

Belaoussoff S., Kevan P.G., Murphy S. and Swanton C. 2003. Assessing tillage disturbance on assemblages of ground beetles (Coleoptera: Carabidae) by using a range of ecological indices. Biodivers. Conserv. 12: 851-882.

Bradley P.N. 1991. Woodfuel, Women and Woodlots, vol. 1. Macmillan Education Ltd, London and Basingstoke 338 pp.

Bradley P.N., Chavangi N. and Van Gelder A. 1985. Development research and energy planning in Kenya. Ambio 14: 228-236.

Colwell R.K. 1997. Estimates: Statistical Estimation of Species Richness and Shared Species from Samples. University of Connecticut, Storrs.

Dougall T.A.G. and Dodd J.C. 1997. A study of species richness and diversity in seed banks and its use for the environmental mitigation of a proposed holiday village development in a coniferized woodland in south east England. Biodivers. Conserv. 6: 1413-1428. 
Gimaret-Carpentier C., Pelissier R., Pascal J.P. and Houllier F. 1998. Sampling strategies for the assessment of tree species diversity. J. Veg. Sci. 9: 161-172.

Gotelli N.J. and Colwell R.K. 2001. Quantifying biodiversity: procedures and pitfalls in the measurement and comparison of species richness. Ecol. Lett. 4: 379-391.

Hayek L.-A.C. and Buzas M.A. 1997. Surveying Natural Populations. Columbia University Press, New York xvi, 563 pp.

Hector A., Schmid B., Beierkuhnlein C., Caldeira C., Diemer M., Dimitrakopoulos P.G., Finn J.A., Freitas H., Giller P.S., Good J., Harris R., Högberg P., Huss-Danell K., Joshi J., Jumpponen A., Körner C., Leadley P.W., Loreau M., Minns A., Mulder C.P., O'Donovan G., Otway S.J., Pereira J.S., Prinz A., Read D.J., Scherer-Lorenzen M., Schulze E.D., Siamantziouras A.D., Spehn E.M., Terry A.C., Troumbis A.Y., Woodward F.I., Yachi S. and Lawton J. 1999. Plant diversity and productivity in European grasslands. Science 286: 1123 1127.

Hill M.O. 1973. Diversity and evenness: a unifying notation and its consequences. Ecology 54: 427431.

Hubbell S.P. 2001. The Unified Neutral Theory of Biodiversity and Biogeography. Princeton University Press, Princeton xiv +375 pp.

ICRAF 1997. ICRAF Medium-Term Plan 1998-2000. International Centre for Research in Agroforestry, Nairobi 77 pp.

Jaetzhold R. 1982. Farm Management Handbook of Kenya: Natural Resources and Farm Management Information vol. iia - West Kenya (Nyanza and Western Provinces). Kenya Ministry of Agriculture, $397 \mathrm{pp}$.

Kindt R. 2001. RenyiAccum. Program to Calculate Average Values and Ranges for the Rényi Diversity Series Associated with Random Site Sequences. International Centre for Research in Agroforestry (ICRAF), Nairobi.

Kindt R. 2004. Biodiversity Analysis Functions for R (Biodiversity.R). ICRAF, Nairobi.

Kindt R., Degrande A., Turyomurugyendo L., Mbosso C., Van Damme P. and Simons A.J. 2001. Comparing species richness and evenness contributions to on-farm tree diversity for data sets with varying sample sizes from Kenya, Uganda, Cameroon, and Nigeria with randomized diversity profiles. In: IUFRO Conference on Forest Biometry, Modelling and Information Science, 26-29 June 2001. University of Greenwich, UK. URL: http://cmsl.gre.ac.uk/conferences/iufro/proceedings/ (last accessed 4-2004).

Kindt R. and Lengkeek A.G. 1999. Tree diversity on farm - use it or lose it. In: National Workshop on Agricultural Biodiversity Conservation, 27-29 January 1999. Intermediate Technology Development Group (ITDG, Nairobi, pp 75-85.

Kindt R., Simons A.J. and Van Damme P. 2004. Do farm characteristics explain differences in tree species diversity among western Kenyan farms? Agroforest. Syst. 63: 63-74.

Legendre P. and Legendre L. 1998. Numerical Ecology. Elsevier Science BV, Amsterdam 853 pp.

Loreau M., Naeem S., Inchausti P., Bengtsson J., Grime J.P., Hector A., Hooper D.U., Huston M.A., Raffaelli D., Schmid B., Tilman D. and Wardle D.A. 2001. Biodiversity and ecosystem functioning: current knowledge and future challenges. Science 294: 804-807.

Loreau M., Naeem S. and Inchausti P. 2002. Biodiversity and Ecosystem Functioning: Synthesis and Perspectives. Oxford University Press, Oxford $304 \mathrm{pp}$.

Magurran A.E. 1988. Ecological Diversity and Its Measurement. Princeton University Press, Princeton, N.Jx, $179 \mathrm{pp}$.

Magurran A.E. and Henderson P.A. 2003. Explaining the excess of rare species in natural species abundance distributions. Nature 422: 714-716.

May R.M. 1975. Patterns of species abundance and diversity. In: Cody M.L. and Diamond J.M. (eds), Ecology and the Evolution of Communities. The Belknap Press of Harvard University Press, Cambridge and London, pp. 81-120.

McGill B.J. 2003. A test of the unified neutral theory of biodiversity. Nature 422: 881-885.

McNeely J.A. and Scherr S.J. 2002. Ecoagriculture: Strategies to Feed the World and Save Wild Biodiversity. Island Press, Washington 323 pp. 
Mishra B.P., Tripathi O.P., Tripathi R.S. and Pandey H.N. 2004. Effects of anthropogenic disturbance on plant diversity and community structure of a sacred grove in Meghalaya, northeast India. Biodivers. Conserv. 13: 421-436.

O'Neill G.A., Dawson I.K., Sotelo-Montes C., Guarino L., Current D., Guariguata M. and Weber J.C. 2001. Strategies for genetic conservation of trees in the Peruvian Amazon basin. Biodivers. Conserv. 10: 837-850.

Purvis A. and Hector A. 2000. Getting the measure of biodiversity. Nature 405: 212-218.

Ricotta C. 2003. On parametric evenness measures. J. Theor. Biol. 222: 189-197.

Ricotta C. and Avena G.C. 2002. On the information-theoretical meaning of Hill's parametric evenness. Acta Biotheor. 50: 63-71.

Rousseau D., Van Hecke P., Nijssen D. and Bogaert J. 1999. The relationship between diversity profiles, evenness and species richness based on partial ordering. Environ. Ecol. Stat. 6: 211-223.

Shaw P.J.A. 2003. Multivariate Statistics for the Environmental Sciences. Hodder Arnold, London ix +233 pp.

Slik J.W.F., Verburg R.W. and Keßler P.J.A. 2002. Effects of fire and selective logging on tree species composition of lowland dipterocarp forest in East Kalimantan, Indonesia. Biodivers. Conserv. 11: 85-98.

Taillie C. 1979. Species equitability: a comparative approach. In: Grassle J.F., Patil G.P., Smith G.K. and Taillie C. (eds), Ecological Diversity in Theory and Practice. International Cooperative Publishing House, Fairland, pp. 51-62.

Tilman D., Reich P.B., Knops J., Wedin D., Mielke T. and Lehman C. 2001. Diversity and productivity in a long-term grassland experiment. Science 294: 843-845.

Tóthmérész B. 1995. Comparison of different methods for diversity ordering. J. Veg. Sci. 6: 283290.

Van Noordwijk M. and Ong C.K. 1999. Can the ecosystem mimic hypotheses be applied to farms in African savannahs? Agroforest. Syst. 45: 131-158.

Zavaleta E.S. and Hulvey K.B. 2004. Realistic species losses disproportionately reduce grassland resistance to biological invaders. Science 306: 1175-1177.

Zilihona I.J.E., Niemela J. and Nummelin M. 2004. Effects of a hydropower plant on Coleopteran diversity and abundance in the Udzungwa Mountains, Tanzania. Biodivers. Conserv. 13: 14531464. 